\title{
Annihilation Radiation Gauge for Relative Density and Multiphase Fluid Monitoring
}

\author{
A. Vidal ${ }^{1}$, G. Viesti ${ }^{2}$, F. Pino ${ }^{1}$, H. Barros ${ }^{1}$, L. Sajo-Bohus $^{1 \mathrm{a}}$ \\ ${ }^{1}$ Universidad Simon Bolivar, Ado 89000, 1080A, Caracas, Venezuela \\ ${ }^{2}$ Dipartimento di Fisica ed Astronomia, Università di Padova, Via Marzolo 8, I-35131Padova, Italy
}

\begin{abstract}
The knowledge of the multi-phase flow parameters are important for the petroleum industry, specifically during the transport in pipelines and network related to exploitation's wells. Crude oil flow is studied by Monte Carlo simulation and experimentally to determine transient liquid phase in a laboratory system. Relative density and fluid phase time variation is monitored employing a fast nuclear data acquisition setup that includes two large volume $\mathrm{BaF}_{2}$ scintillator detectors coupled to an electronic chain and data display in a LabView ${ }^{\circledR}$ environment. Fluid parameters are determined by the difference in count rate of coincidence pulses. The operational characteristics of the equipment indicate that $2 \%$ deviation in the CCR corresponds to a variation, on average, of $20 \%$ in the fraction of liquid of the multiphase fluid.
\end{abstract}

\section{Introduction}

During fluid transport the knowledge of pressure drop, liquid hold-up, and flow pattern is important since two-phase flow occurs and may damage pumping machinery inserted in pipelines or in deep oil wells. It is estimated that more than one-half the natural gas flows in two-phase flow lines and it may induce deterioration and ageing of equipment. In Venezuela as well as in other countries most wells produce oil accompanied by some gas, water and solid material that affects strongly the operational life time of extracting pumps positioned often at more than 3 thousand feet deep in the soil. Therefore a method for predicting fluid characteristics before intake could extend the oil well operational interval [1-3]. Although extensive research in two-phase flow has been conducted during the past 25 years, new techniques are expected to improve oil business machinery. The determination of transient liquid phase and dynamical behaviour of gas-liquid flow is conveniently studied in a laboratory assembly. Preliminary technical details of a system designed and tested to provide on-line fluid multiphase monitor employing annihilation radiation gauge for oilfield application has been proposed earlier [4]. Here an extension of the study is given, namely, the operational characteristics of the equipment, its stability and limitations are reported for gas - liquid (oil, water, glycerine and ethyl alcohol) fraction measurements which conducted in a purpose made test manifolds nodes assembled in laboratory under simulated field conditions. Monte Carlo simulations have been employed to improve the system efficiency.

\footnotetext{
${ }^{a}$ Corresponding author: sajobohus@gmail.com
} 


\section{Operational Principle}

The emitted positron during the sodium isotope decay, interacts with electrons in the absorbing media experimenting the annihilation reaction resulting in the emission of two $511 \mathrm{keV}$ photons travelling in opposite direction. Some of those interact with the scintillator detectors positioned in a face to face geometry and some of the generated pulses are recorded in coincidence (see Fig. 1). By positioning a pipe filled with known fluids between these two opposed detectors, it is possible to monitor the phase time-variation by the number of pulses in coincidence. In fact the coincidence count rate $(\mathrm{CCR})$ is proportional to the fluid composition i.e. mainly to the different percentage of gas, water and oil. Displayed data, handled in a LabView ${ }^{\circledR}$ environment, are indicative of the transient fluid allowing for on line monitoring and therefore for prompt technical intervention on pump machinery power when required.

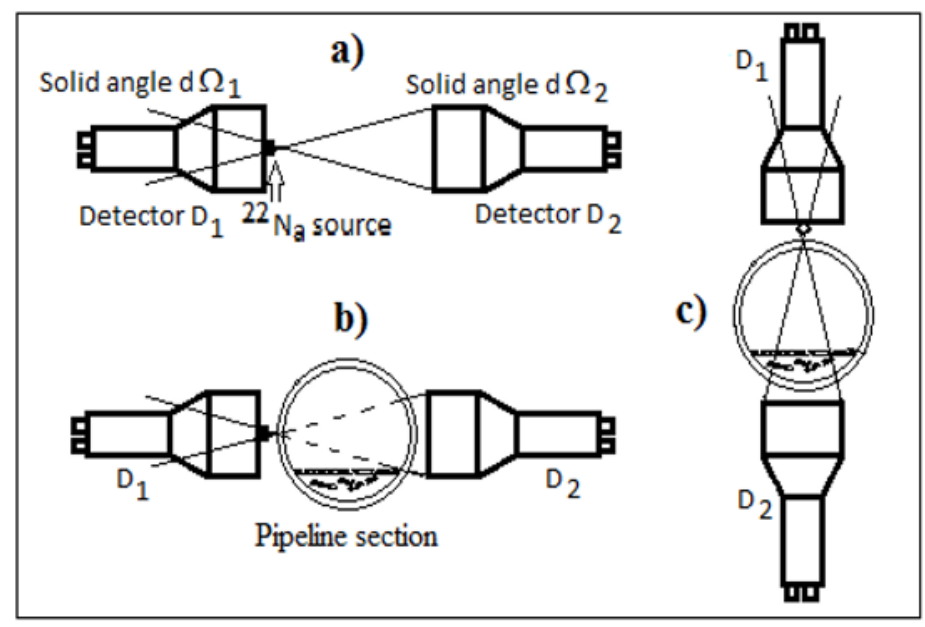

Figure 1. Scheme of the monitoring system geometry. a) Shows the positron source placed on D1 and the detectors in horizontal position. b) Same as a) but with the pipeline. c) Detectors in vertical position.
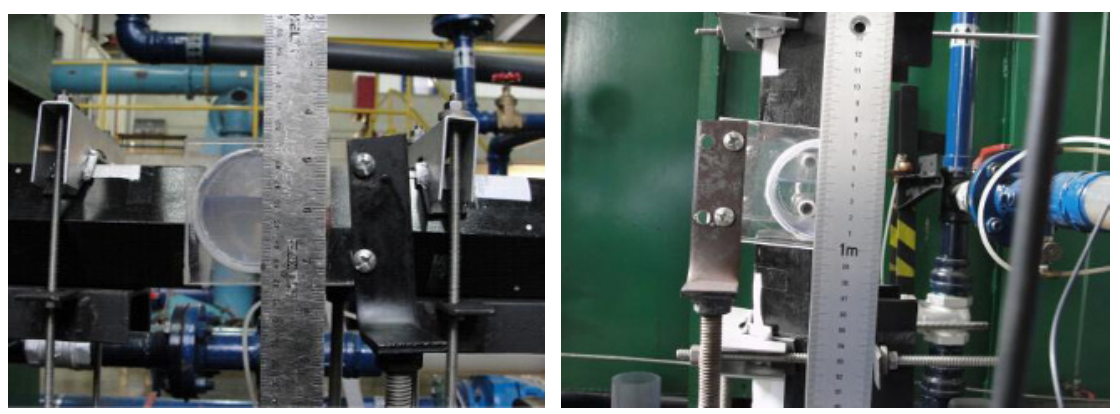

Figure 2. (Left) Detectors in horizontal position showing the fluid level (water and air). (Right) Detectors in vertical position showing again the fluid level.

\section{Experimental Set-up}

The nuclear monitoring device consists of a $1 \mu \mathrm{Ci}$ positron emitting source $\left({ }^{22} \mathrm{Na}\right)$ and two large volume (3"x3") scintillator detectors coupled to a standard electronic chain. The acquired data are displayed and recorded on an interface made in LabView ${ }^{\circledR}$ environment. As mentioned, fluid parameters are determined by the difference in count rate of coincidence pulses [5]; setting reference 
values it is possible to take the decision to modify operating on-line pumps parameters in order to avoid abrupt changes in fluid composition, which cause damage in the mechanical rotating part.

The operational characteristics of the nuclear unit were determined on laboratory test set-up in which the fluid composition, temperature and pressure could be changed. Along the piping system a PVC transparent pipe of $63 \mathrm{~mm}$ diameter and $150 \mathrm{~mm}$ length, was conveniently positioned between the two scintillator detectors (located vertically or horizontally as shown in Fig. 2). This section of pipeline was filled with previously measured volumes of various different liquids (water, oil, glycerine and ethyl-alcohol). The metallic rule in Fig. 2 is used to monitor the fluid level. For each volume occupied by the fluid the corresponding gamma sensors data were recorded for further analysis. Time variation of the measured count rate was taking into account by doing an average over fixed time $(1 \mathrm{~min})$ interval set $(1 \mathrm{~s})$ by the operator.

\section{Results and Discussion}

In order to determine the monitoring device response, the coincidence count rate in function of the fraction of liquid related to a two-phase fluid (air-water and air-ethyl alcohol) is given in Fig. 3, where the geometry of the detectors was alternated between vertical (Fig. 3-left) and horizontal position (Fig. 3-right) with respect to the pipeline axis. We found that the horizontally set up has a lower performance, therefore the best geometry is clearly the vertical one. For that set up, to determine the fluid composition or the average density, the coincidence count rate was monitored for several twophase fluids i.e. mixture of gas (air), water, oil and others fluid of interest, in a well defined percentage. Fig. 4-left shows the results for the air-water and air-oil fluid composition. It is worth noting that a good discrimination between these two types of fluid is provided. Regarding the others two-phase fluids, Monte Carlo simulations were performed and the results are given in Fig. 4-right, which show that the discrimination between the fluids mixture could be extended to air-glycerine and air-ethyl alcohol. The range of the operational parameters for the studied two-phase fluids, with 0$100 \%$ fraction of liquid, will be well distinguished by the system. The standard deviation of the coincidence count rate is $2 \%$. It was found by acquiring data during 1 minute every $1 \mathrm{~s}$. It is observed that $2 \%$ deviation in the CCR corresponds to a variation, on average, of $20 \%$ in the fraction of liquid of the two-phase fluid (see Fig. 4-left). To improve knowledge on the system characteristics, Monte Carlo simulations for a pipe of $63 \mathrm{~mm}$ outer diameter were made and the results were compared with the experimental values.
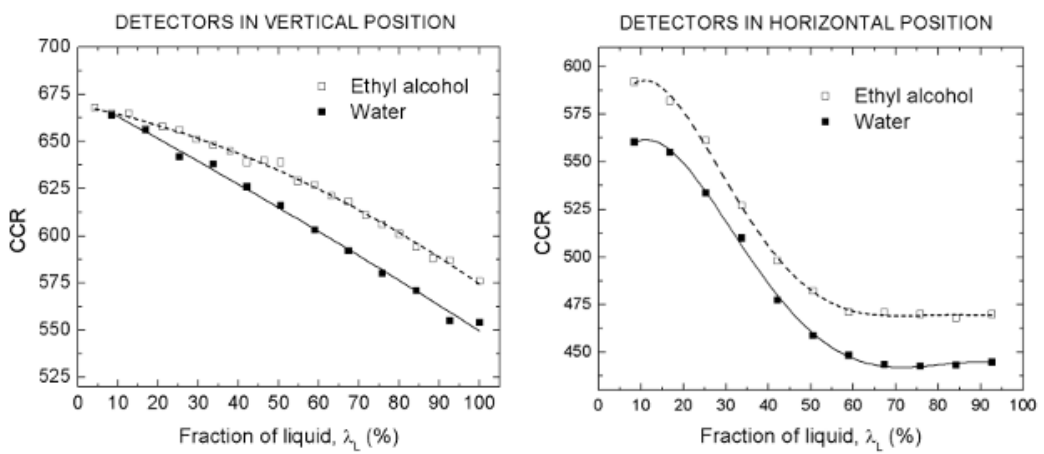

Figure 3. CCR for two-phase fluids (air-water and air-ethyl alcohol) with different fractions of liquid when detectors are in vertical (left) and in horizontal (right) position. Uncertainties of the CCR are $2 \%$.

\section{Monte Carlo Modelling}

Monte Carlo simulation of the counting system was performed with PENELOPE version 2008 code, which is a widely validated code for the transport of photons, electrons and positrons. The 
simulation consisted in follow the history of only "G2" which is the $511 \mathrm{keV}$ gamma ray that goes through the pipeline (towards D2), because we assumed that "G1", the other gamma ray that goes towards D1, is always detected when G2 get activated, see Fig 1. By the count rate in D2 we could estimate the experimental coincidence rate for different two-phase fluids. Resulting data were used to extend the experimental results. Besides, it was possible to deduce technical improvement on the pipedetector geometry, the minimum require source strength and best counting efficiency.
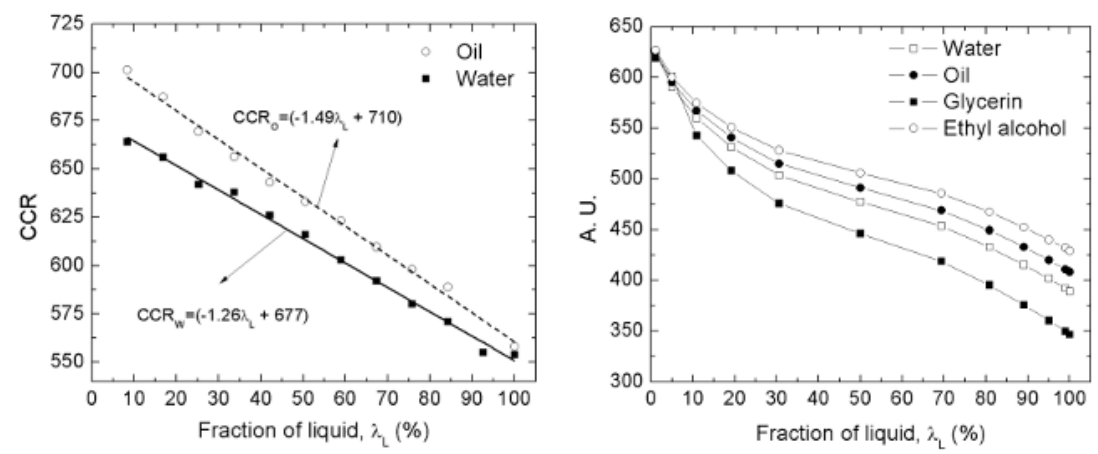

Figure 4. (Left) Comparison of the coincidence count rate between air-water and air-oil two-phase fluids in different percentages. (Right) Monte Carlo simulations of several two-phase fluids.

In Fig 4-right the Monte Carlo results are plotted. The discrimination between air-water and air-oil is similar to that observed with the experimental measurements, suggesting the possibility at least in principle to extend the studies to other of two-phase fluids mixture (air-glycerine and air-ethyl alcohol). In fact, it gives us the possibility to extend the technique to multi phase fluids in that gas, liquid and solid phases take place in different percentage.

\section{Conclusions}

To respond to an urgent request to improve national oil production, a nuclear monitoring device for two-phase fluid was assembled. Gas-liquid fluid was studied to determine transient liquid phase in a laboratory system. Relative density and fluid phase time variation were determined by nuclear technique. The operational characteristics of the equipment indicate that a $2 \%$ deviation in the CCR corresponds to a variation, on average, of $20 \%$ in the fraction of liquid of the multiphase fluid. The system performances comply with requirements imposed for field processing equipment.

\section{Acknowledgement}

Thanks to the Dipartimento di Fisica ed Astronomia dell' Università di Padova for the unconditional technical support and for the help given by M. Caldogno at the early stage of the project. The financial support given by the Energy Conversion Group (USB) is acknowledged.

\section{References}

1. P. Spedding, D. Spence, J. Multiphase Flow, 245-280, (1993).

2. S. Tjugum, G. Johansen, M. Holstad. Radiation Physics and Chemistry, 61 (2001).

3. R. Hoffmann, G. Johnson. Flow Measurement and Instrumentation 22 (2011).

4. A. Vidal, et. al. American Institute of Physics, Conf., Proceedings. 1423, (2011).

5. G. Viesti, et al. Nuclear Inst. and Methods in Physics Research, A, 606, (2009). 